Eull. Mater. Sci., Vol. 2, Number 2, May 1980, pp. 83-89. C Printed in Iadia.

\title{
Characterisation of bubble materials
}

\author{
M I PATNI, OM PARKASH* and D BAHADUR* \\ Materials Science Centre, Indian Institute of Technology, Powai, Bombay 400076 , \\ India \\ - Advanced Centre for Materials Science, Indian Institute of Technology, \\ Kanpur 208 016, India \\ MS received 21 February 1980
}

\begin{abstract}
The rapid development of magnetic bubble technology has required growth to exacting specifications of a large number of magnetic films of garnets and some amorphous binary and ternary alloys of rare-earth and transition metals. The characterisation of these films is an essential part in the search for newer materials which hold out promise for better device performance and cost viability. Many methods of films characterisation have been reported from time to time. By and large, these methods can be divided into two groups : one, bulk measurements made on the film and two, measurements made on the domains. We have attempted to collate and briefly introduce various techniques to characterise magnetic bubble materials in this review.
\end{abstract}

Keywords. Magnetic bubbles; magnetisation; mobility; coercivity, crystal quality.

\section{Introduction}

Magnetic bubbles are cylindrical domains which may be created in thin films of appropriate magnetic materials having uniaxial anisotropy. In a magnetic field gradient these domains can acquire substantial velocities, a feature which makes them attractive as a possible memory device. The rapid development of magnetic bubble technology requires growth to exacting specifications of a large number of magnetic films. The basis of many of these methods is the strip-domain theory of Kooy and Enz (1960) and the stability criteria as given by Thiele (1970).

The static behaviour of the bubbles is completely specified by the three parameters namely thickness $h$, saturation magnetisation $4 \pi M$, and characteristic length $l$. The characteristic length $l$ is a function of the magnetisation and is given by $\sigma_{w} / 4 \pi M_{*}^{*}$ where $\sigma_{*}$ is the wall energy. The quality factor $Q$ which determines the ability of a medium to support magnetic bubbles is the ratio of the induced anisotropy constant $K_{\mathrm{u}}$ and $M_{a}^{2}$, and should be greater than unity.

The dynamic behaviour of the bubbles can be characterised by the bubble mobility $\mu$. The concept of mobility can be applied to a bubble domain in translation as long as a simple linear relation exists between the applied freld 
and the bubble velocity. Below a threshold field (the so-valled coercive field $\boldsymbol{H}_{\boldsymbol{c}}$ ) no appreciable motion occurs. The linear relationship between the velocity and driving force pertains until a critical velocity is reached when a breakdown occurs (dynamic conversion effects) (Argyle et al 1971).

The complete characterisation of a magnetic bubble film would require the determination of chemical composition, concentration of defects, as well as $h, 4 \pi M_{s}, l, K_{u}, H_{s}$ and $\mu$.

The first important step after film preparation is the determination of its composition since all the parameters critically depend on it. This is possible by radioactive isotope tracer technique (Stacy et al 1972), electron microprobe and spectrophotometric analysis (Cronemeyer et al 1971). Depth profiling technique using sputter ion etching $x$-ray fluorescence and Auger elemental analysis have also been used for the same (Hoffman and Winkler 1979).

\section{Measurement of parameters}

\subsection{Crystal quality}

It is essential to find the concentration of defects. The following techniques are commonly used to detect defects in films :

(i) A simple though destructive technique is to etch the film at an appropriate temperature (Stein 1973).

(ii) X-ray topography is utilised to get a point-to-point image of the diffracting power of the sample on a photographic plate (Stacy and Enz 1972; Glass 1972). The intensity diffracted by the deformed crystal differs from that of a perfect crystal and a defect map is obtained. By this method, it is possible to determine the defects in the film and the substrate (Keig 1972).

(iii) A bubble is generated at one conductor and made to propagate to another by means of a three phase current pulse sequence (Pierce 1974). Defects appear as stationary bubbles. A time exposure photograph decorates the defect region.

(iv) A single bubble is raster scanned through the area of the film and defect is detected by the distortion in the bubble area (Shumate 1971).

(v) Spatial filtering allows first and higher order diffracted waves to pass the back focal plane to produce an instantaneous map of bubble defects (Henry 1976; Maartense et al 1978).

(vi) Transmission electron microscope (Lorentz mode) determines the number of bloch lines. This is essential as the dynamic character of the bubbles depends on the number of blowh lines (Grundy 1977). The transmission electron microscopy is particularly good for smaller bubbles where optical microscopy fails (Bahadur and Rai 1980).

(vii) The internal strain, which depends on the lattice mismatch, is obtained by measuring the separation angle between two peaks due to the film and substrate by means of a double crystal diffractometer (Hattanda and Takeda 1973).

(viii) When the inplane field drops below the nucleation field, the shape and size of the domain also gives information about magnetic imperfections (Akselrad 1972). 


\subsection{Thickness}

One of the most important parameters of a bubble domain layer is its thickness $h$ as it predetermines the diameter of the magnetic bubble. The most popular method for the determination of $h$ uses interferometric technique, particularly for garnets, because the film and the substrate show good transparency for wavelength from $0.5 \mu \mathrm{m}$ upto about $5 \mu \mathrm{m}$. Interference occurs between rays internally reflected from the air-film interface and the film-substrate interface. By counting the number of maxima in the transmitted intensity as a function of the wavelength of the incident light, $h$ can be measured (Ghez and Giess 1973). Inaccuracies result, however, from not knowing the value of the refractive index of the film as a function of wavelength and from any lead diffused into the substrate during the film growth. The total error can be upto $4 \%$.

Thickness can also be determined by using a large metal sphere $(50 \mathrm{~mm}$ in diameter) coated with diamond polishing compound to grind a special hole just through the film and measuring the radii of the concentric circles of the film layer (Stacy et al 1972). The flatness of the film can be determined by studying the spin wave resonance line width (Hockstra and Rokertson 1975). Thickness and optical index of refraction of thin bubble film $(<3 \mu \mathrm{m})$ can be determined using ellipsometry (Mier et al 1979). This eliminates the uncertainties associated with the interferometric technique.

\subsection{Saturation magnetisation and characteristic length}

Characteristic length $(l)$ is determined from a knowledge of saturation magnetisation $\left(M_{s}\right)$ and wall energy as described in $\S 1$. Techniques commonly used for evaluating $4 \pi M_{s}$ are the vibrating sample magnetometer (Schreurs and Borelli 1972) and the pendulum magnetometer (Enz et al 1962).

A magneto-optic bench exploiting the optical Faraday or Kerr effect is used for determination of $l$ and $4 \pi M_{s}$ simultaneously. Light from a $\mathrm{He}-\mathrm{Ne}$ laser or a xenon-arc lamp is polarised and made incident on the film. Domains can be viewed through a suitably crossed analyser and domain width or bubble diameter can be measured using a frlar eyepiece (Josephs 1972). The most popular measurements are the following:

(i) The bubble diameter $d$ is measured as a function of a DC magnetic field $H$ applied normal to the film. A graph is plotted of

$$
H d \text { vs } d[1+(3 d / 4 h)]^{-1}
$$

from which $4 \pi M_{s}$ and $l$ are determined. The value of $4 \pi M_{s}$ (slope of the graph) can be determined fairly accurately ( $15 \%$ ) but the value of $l$ (intercept) can have a sizable error (土 40\%) (O' Dell 1974).

(ii) A straight boundary wall is produced with a uniform magnetic field gradient. As the gradient is decreased below a critical level the wall becomes sinusoidal. The wavelength of the sinussoid depends on $l$. From the value of the critical field gradient one can also determine $4 \pi M_{\mathrm{a}}$. The estimated errors in the determination are : $4 \pi M_{*}- \pm 10 \%$ and $l- \pm 7 \%$ (Rijnierse and de Leeuw 1973).

(iii) A demagnetised stripe domain pattern is obtained by applying a large AC field and slowly reducing it to zero. The zero field stripe-width is measured 
over as many stripes as possible. One then measures the critical field for bubble collapse. From these measurements both $l( \pm 4 \%)$ and $4 \pi M$. (土 $2 \%$ ) are calculated (Fowlis and Copeland 1972a).

(iv) The diameters of the bubble and the bias field at collapse and run-out are measured. Both $4 \pi M_{s}( \pm 5 \%)$ and $l( \pm 10 \%)$ can then be determined (O'Dell 1974).

The above techniques depend on the visual observation of bubbles and are therefore susceptible to operator errors. Moreover, measurements are to be carried out on single bubbles and so one must ensure that the bubble is not pinned and is sufficiently far away from other bubbles.

\subsection{Anisotropy constant}

(i) The most direct method to measure $K_{w}$ is by using the torque magnetometer. The commercial instrument can give the average value of $K_{w}$ for the whole film (Sery and Irons 1973).

(ii) A large DC field is applied parallel to the film and an AC field is applied perpendicular to it. Polarised light transmitted by the film is synchronously detected to give a signal proportional to the susceptibility. From the high field behaviour of susceptibility the anisotropy constant can be determined with an accuracy of $\pm 10 \%$ (Shumate et al 1973).

(iii) A magnetic field is applied in the plane of the film and is slowly increased in magnitude till the contrast between adjacent domains disappears (Smith et al 1972).

(iv) By studying the change in the resonance field (by FMR) with DC field parallel and perpendicular both $4 \pi M_{s}$ and $K_{w}$ can be evaluated (Cronemeyer 1973). It may be added that resonance linewidth is related to the damping parameter which in turn is related to the mobility (Vella-Coleiro 1972).

\subsection{Bubble mobility}

This is probably the most difficult parameter to measure and in literature there are a large number of techniques for its evaluation. The more popular ones are listed below :

(i) The measurement is carried out on a single bubble. The bias field is increased by applying a square pulse, causing the bubble to start shrinking. If the pulse lasts long enough the bubble shrinks below the collapse radius so that after the end of the pulse it collapses. By measuring the length of the pulse that just collapses the bubble as a function of pulse ampli. tude, $\mu$ can be deduced. The pulse width is usually of the order of nanoseconds and so a correction must be applied to take into account the response time of the measuring instrument. The method, though tedious is quite simple and yields accuracies of $\pm 10 \%$ (Josephs and Stein 1973).

(ii) Another method observes the response of the bubble in a sinusoidal external field. As in the previous method the analysis is difficult as the driving force on the wall is time-varying. Also in the case of garnets one 
needs to employ high frequencies. However, the method has been used with advantage in orthoferrites, or for large bubble (Rossol 1969).

(iii) In order to avoid the problem of time varying velocities the permalloy disk technique has been used where the motion of a bubble around a permalloy disk is observed stroboscopically. This method has been used for large bubbles but is not readily adaptable for measurements on garnets (Jones et al 1979).

(iv) A method which has been commonly used for garnets is to observe the motion of the bubble in a DC field gradient. A narrow rise time pulse current is used in a strip conductor to translate the bubble. The bubble position is measured before and after the pulse and its average velocity computed by dividing the displacement by the pulse width. A plot of the translational velocity versus difference in bias field gives the mobility (Kryder and $\mathrm{Hu}$ 1973).

(v) In another method, measurements are made on a stripe domain. The experiment is to measure the phase shift between the domain wall displacement and the applied alternating field drive. Observations are made using the Faraday effect and calibration has to be done using a standard (terbium aluminium garnet). The results give an accuracy of $\pm 8 \%$ (Fowlis and Copeland 1972b).

(vi) A demagnetised sample is subjected to a repetitive pulse field. The resulting stripe domain wall displacement is observed via a sampling technique. The method is quick and simple with an accuracy of $\pm 15 \%$ (Vella-Coleiro 1972).

\subsection{Coercive field}

(i) Generally, coercivity is determined by studying the motion of stripe domains in a demagnetised sample in response to a pulse or AC magnetic field normal to the sample. The amplitude of the response is plotted versus the applied field. This plot is roughly linear at large fields (the slope being the mobility) and the intercept on the field axis is taken to be the coercivity (Josephs 1972).

The accuracy of the method can be considerably increased if one compares the in-phase and quadrature fundamental frequency components of the domain wall motion in response to an AC field. Mean coercivities as low as 0.01 oersteds can be measured (Clover and Cutler 1972).

(ii) Probably, the most direct method is to study the hysteresis loop using an optical hysteriograph and directly measuring the coercivity (Shumate et al 1973).

(iii) An interesting method proposed is to measure the equilibrium distance to which two bubbles get separated after being forced together and the force abruptly removed. The method is simple (accuracy $\pm 15 \%$ ) but not very suitable for small diameter bubbles (Bobeck et al 1969).

\section{Conclusion}

In writing this review we have not attempted to critically evaluate all the papers in the literature. The various methods mentioned in this review are clearly an 
indication of the immense activity in this area and we have just attempted to briefly introduce the readers with these techniques.

In recent years, several workers have used some newer techniques which are worth mentioning. Recently light scattering from spin waves has been used to determine anisotropy constant and exchange constant (Grundberg 1979). High speed photography has been used to study the rupture of domains, dynamic distortion of stripe and bubble domains (Randoshkin 1978). Scanning electron microssope has been exploited to study the stripe domain width and so on (Jones and Dunk 1977) and so on. For thin bubble materials, microwave resonance (Mier et al 1976) and audiofrequency susceptibility measurement (Maartense et al 1978) seem to be superior to other methods (Mier et al 1979).

\section{Acknowledgements}

The authors are thankful to Dr C M Srivastava for many helpful discussions.

\section{References}

Akselrad A 1972 AIP Conf. Proc. 10408

Argyle B E, Slonczewski J C and Mayadas A F 1971 AIP Conf. Proc. 5175

Bahadur D and Rai K N 1980 Commuunicated to Mat. Res. Bull.

Bobeck A H, Fischer R F, Perneski A J, Remeika J P and Van Uitert L G 1969 IEEE Trans. $M A G-5544$

Clover R B and Cutler L A 1972 AIP Conf. Proc. 18388

Cronemeyer D C, Giess E A, Klokholm E, Argyle B E and Plaskett T S 1971 AIP Conf. Proc. 5 115

Cronemeyer D C 1973 AIP Conf. Proc. 1885

Enz U, Fast J F, Van Houten S and Smit J 1962 Philips Res. Rep. 17451

Fowlis D C and Copeland J A 1972a AIP Conf. Proc. 5240

Fowlis D C and Copeland J A 1972b AIP Conf. Proc. 10393

Ghez R and Giess E A 1973 Mat. Res. Bull. 831

Glass H L 1972 Mat. Res. Bull. 7385

Grundberg P 1979 J. Magn. Magn. Mater. 13181

Grundy P J 1977 Contemp. Phys. 1647

Hattanda T and Takeda A 1973 Jpn. J. Appl. Phys. 121104

Henry R D 1976 Mat. Res. Bull. 111285

Hoffmann H and Winkler R 1979 J. Magn. and Magn. Mater. 1389

Hockstra B and Robertson J M 1975 AIP Conf. Proc. 34178

Jones $C A$ and Dunk P 1977 Physica $B$ and $C$ 86-88 1345

Jones C A, Stroomer M V C, Voegeli O and Friedlaender F J 1979 Trans. IEEE MAG-15 926

Josephs R M 1972 AIP Conf. Proc. 10286

Josephs R M and Stein B F 1973 AIP Conf. Proc. 18227

Keig G A 1972 AIP Conf. Proc. 10237

Kooy C and Enz U 1960 Philips Res. Rep. 157

Kryder M H and Hu H L 1973 AIP Conf. Proc. No. 18213

Maartense I, Searle C W and Mier M G 1978 J. Appl. Phys. 491882

Mier M G, Swenson H W S and Wigen P E 1976 AIP Conf. Proc. No. 34178

Mier M G, Blasingame J M, Searle C W, Maartense I, Wigen P E, Omaggio J P, Globe M P and Limaya R S 1979 J. Appl. Phys. 502185

O'Dell T H 1974 Magnetic bubbles (London : Macmillan) pp 75, 78

Pierce R D 1974 J. Cryst. Growth 27299

Randoshkin V V, Ivanov L P and Telasnin R V 1978 Sov. Phys. JETP 48486

Rijnierse P J and de Leeuw F H 1973 AIP Conf. Proc. 18119 
Rossol F C 1969 J. Appl. Phys. 401082

Schreurs J W H and Borelli N F 1972 J. Appl. Phys. 433882

Sery R S and Irons H R 1973 AIP Conf. Proc. 1890

Shumate P W 1971 J. Appl. Phys. 421274

Shumate P W, Smith D H and Hagedorn F B 1973 J. Appl. Phys. 44449

Smith A B, Kestigian M and Bekebrede W R 1972 AIP Conf. Proc. 10309

Stacy $\mathrm{W}$ T and Enz U 1972 IEEE Trans. MAG-8 269

Stacy W T, Janssen M M, Robertson J M and Van Hout M J G 1972 AIP Conf. Proc. 10314

Stein B F 1973 AIP Conf. Proc. 1848

Thiele A A 1970 J. Appl. Phys. 411139

Vella-Coleiro G P 1972 AIP Conf. Proc. 10424 